\title{
DENSITY PROPERTIES FOR FRACTIONAL SOBOLEV SPACES
}

\author{
Alessio Fiscella, Raffaella Servadei and Enrico Valdinoci \\ Università di Milano, Dipartimento di Matematica \\ Via Cesare Saldini 50, 20133 Milano, Italy; alessio.fiscella@unimi.it \\ Università della Calabria, Dipartimento di Matematica e Informatica \\ Ponte Pietro Bucci 31 B, 87036 Arcavacata di Rende (Cosenza), Italy; servadei@mat.unical.it \\ Università di Milano, Dipartimento di Matematica \\ Via Cesare Saldini 50, 20133 Milano, Italy \\ and Weierstraß-Institut für Angewandte Analysis und Stochastik \\ Mohrenstraße 39, 10117 Berlin, Germany; enrico.valdinoci@wias-berlin.de
}

\begin{abstract}
Aim of this paper is to give the details of the proof of some density properties of smooth and compactly supported functions in the fractional Sobolev spaces and suitable modifications of them, which have recently found application in variational problems. The arguments are rather technical, but, roughly speaking, they rely on a basic technique of convolution (which makes functions $C^{\infty}$ ), joined with a cut-off (which makes their support compact), with some care needed in order not to exceed the original support.
\end{abstract}

\section{Introduction}

1.1. Motivations. Recently, in the literature a deep interest was shown for nonlocal operators, thanks to their intriguing analytical structure and in view of several applications in a wide range of contexts, such as the thin obstacle problem, optimization, finance, phase transitions, stratified materials, anomalous diffusion, crystal dislocation, soft thin films, semipermeable membranes, flame propagation, conservation laws, ultra-relativistic limits of quantum mechanics, quasi-geostrophic flows, multiple scattering, minimal surfaces, materials science and water waves: see for instance $[3,4,5,6,8,9,10,11,14,15,16,20,21,22,23,24,27,29,30,31,32,33]$ and references therein. One of the typical models considered is the equation

$$
\begin{cases}(-\Delta)^{s} u=f(x, u) & \text { in } \Omega, \\ u=0 & \text { in } \mathscr{C} \Omega,\end{cases}
$$

where $s \in(0,1)$ is fixed, $\Omega \subseteq \mathbf{R}^{n}$ is an open set, $\mathscr{C} \Omega:=\mathbf{R}^{n} \backslash \Omega$ is its complementary set, $-(-\Delta)^{s}$ is the fractional Laplace operator which (up to normalization factors)

doi:10.5186/aasfm.2015.4009

2010 Mathematics Subject Classification: Primary 46E35, 35A15, 35S15; Secondary 47G20, $45 \mathrm{G} 05$.

Key words: Fractional Sobolev spaces, density properties, integrodifferential operators, fractional Laplacian.

The second author was supported by the MIUR National Research Project Variational and Topological Methods in the Study of Nonlinear Phenomena and by the GNAMPA Project 2014 Geometric and Analytic Properties for Nonlocal Problems, while the third one by the MIUR National Research Project Nonlinear Elliptic Problems in the Study of Vortices and Related Topics and the FIRB project A\&B (Analysis and Beyond). All the authors were supported by the ERC grant $\varepsilon$ (Elliptic Pde's and Symmetry of Interfaces and Layers for Odd Nonlinearities). 
may be defined as

$$
-(-\Delta)^{s} u(x)=\int_{\mathbf{R}^{n}} \frac{u(x+y)+u(x-y)-2 u(x)}{|y|^{n+2 s}} d y
$$

for $x \in \mathbf{R}^{n}$ (see $[12,28]$ and references therein for further details on the fractional Laplacian) and the right-hand side $f$ is a function satisfying suitable regularity and growth conditions.

Problem (1.1) has a variational structure and the natural space where finding solutions for it is the fractional Sobolev space $H^{s}\left(\mathbf{R}^{n}\right)$ (see $\left.[1,12]\right)$. Note that in (1.1) the homogeneous Dirichlet datum is given in $\mathscr{C} \Omega$ and not simply on $\partial \Omega$, as it happens in the classical case of the Laplacian, consistently with the nonlocal character of the operator $(-\Delta)^{s}$.

In order to study (1.1) through variational techniques it is important to encode the 'boundary condition' $u=0$ in $\mathscr{C} \Omega$ in the weak formulation of the problem. For this the usual fractional Sobolev space is not enough. At this purpose, for any fixed $s \in(0,1)$ and $p \in[1,+\infty)$ we consider the kernel $K: \mathbf{R}^{n} \backslash\{0\} \rightarrow(0,+\infty)$ such that

$$
m K \in L^{1}\left(\mathbf{R}^{n}\right) \text {, where } m(x)=\min \left\{|x|^{p}, 1\right\} ;
$$

there exists $\vartheta>0$ such that $K(x) \geqslant \vartheta|x|^{-(n+s p)}$ for any $x \in \mathbf{R}^{n} \backslash\{0\}$.

It is easily seen that a model for $K$ is given by the singular kernel $K(x)=|x|^{-(n+s p)}$ which gives rise to the fractional Laplace operator $-(-\Delta)^{s}$ defined in $(1.2)$ when $p=2$. In this framework we introduce the space $X^{s, p}\left(\mathbf{R}^{n}\right)$ as the linear space of Lebesgue measurable functions $g$ from $\mathbf{R}^{n}$ to $\mathbf{R}$ such that the following quantity is finite:

$$
\|g\|_{L^{p}\left(\mathbf{R}^{n}\right)}+\left(\int_{\mathbf{R}^{n} \times \mathbf{R}^{n}}|g(x)-g(y)|^{p} K(x-y) d x d y\right)^{1 / p} .
$$

Then, we define $X_{0}^{s, p}(\Omega)$ as the space of functions $g \in X^{s, p}\left(\mathbf{R}^{n}\right)$ that vanish a.e. in $\mathscr{C} \Omega$.

Clearly, the quantity in (1.5) defines a norm on $X^{s, p}\left(\mathbf{R}^{n}\right)$ and $X_{0}^{s, p}(\Omega)$ : such norm will be denoted simply by $\|\cdot\|$.

We observe that the space $X_{0}^{s, p}(\Omega)$ is similar to, but different from, the usual fractional Sobolev space $W^{s, p}(\Omega)$, which is endowed with the norm

$$
\|g\|_{W^{s, p}(\Omega)}:=\|g\|_{L^{p}(\Omega)}+\left(\int_{\Omega \times \Omega} \frac{|g(x)-g(y)|^{p}}{|x-y|^{n+p s}} d x d y\right)^{1 / p} .
$$

Even in the model case in which $K(x)=|x|^{-(n+s p)}$, the norms in (1.5) and (1.6) are of course not the same. For further details we refer to $[12,25,26]$ and to the references therein.

1.2. Main results of the paper. As well-known, the natural functional space associated to the classical Laplace setting, i.e. problem (1.1) with $s=1$, is the Sobolev space $H_{0}^{1}(\Omega)$. When $\partial \Omega$ is sufficiently regular, such space may be equivalently defined as the set of functions that belong to $L^{2}(\Omega)$ together with their weak derivatives of order one and that vanish along $\partial \Omega$ in the trace sense, or as the closure of $C_{0}^{\infty}(\Omega)$ in the norm of $H^{1}(\Omega)$ (see, for instance, [1]).

Aim of this paper is to study the density properties of the space $X_{0}^{s, p}(\Omega)$, in order to establish a result similar to the one known in the classical case. Precisely, 
we investigate the relation between the spaces $X_{0}^{s, p}(\Omega)$ and $C_{0}^{\infty}(\Omega)$. Since $X_{0}^{s, p}(\Omega)$ is a space of functions defined in $\mathbf{R}^{n}$, in this context we denote by $C_{0}^{\infty}(\Omega)$ the space

$$
C_{0}^{\infty}(\Omega)=\left\{g: \mathbf{R}^{n} \rightarrow \mathbf{R}: g \in C^{\infty}\left(\mathbf{R}^{n}\right), \operatorname{Supp} g \text { is compact and Supp } g \subseteq \Omega\right\},
$$

where $\operatorname{Supp} g=\overline{\left\{x \in \mathbf{R}^{n}: g(x) \neq 0\right\}}$. Notice that if $g \in C_{0}^{\infty}(\Omega)$, then $\operatorname{Supp} g$ is always bounded, even if here and in the sequel we do not assume that $\Omega$ is bounded.

Indeed, the first case we deal with is when $\partial \Omega$ is a graph of a continuous function (notice that, in this case, neither $\Omega$ nor $\partial \Omega$ are bounded):

Definition 1. The open set $\Omega \subseteq \mathbf{R}^{n}$ is a hypograph if there exists a continuous function $\xi: \mathbf{R}^{n-1} \rightarrow \mathbf{R}$ such that, up to a rigid motion,

$$
\Omega:=\left\{\left(x^{\prime}, x_{n}\right) \in \mathbf{R}^{n-1} \times \mathbf{R}: x_{n}<\xi\left(x^{\prime}\right)\right\} .
$$

In this framework our density result can be stated in this way:

Theorem 2. Let $\Omega \subseteq \mathbf{R}^{n}$ be a hypograph. Then, for any $u \in X_{0}^{s, p}(\Omega)$ there exists a sequence $\rho_{\varepsilon} \in C_{0}^{\infty}(\Omega)$ such that $\left\|\rho_{\varepsilon}-u\right\| \rightarrow 0$ as $\varepsilon \rightarrow 0$. In other words, $C_{0}^{\infty}(\Omega)$ is a dense subspace of $X_{0}^{s, p}(\Omega)$.

Remark 3. The sequence of function $\rho_{\varepsilon}$ in Theorem 2 also enjoys the additional property of being supported in the vicinity of the support of the original function $u$. More precisely, fixed any $\gamma>0$ there exists $\varepsilon_{\gamma}>0$ such that for any $\varepsilon \in\left(0, \varepsilon_{\gamma}\right]$ one has that

$$
\operatorname{Supp} \rho_{\varepsilon} \subseteq \operatorname{Supp} u+B_{\gamma} .
$$

The next case that we consider is when $\Omega$ is a domain with continuous boundary, according to the following definition (see e.g. [19, Definition 3.28]):

Definition 4 . The open set $\Omega \subseteq \mathbf{R}^{n}$ is a domain with continuous boundary $\partial \Omega$ if the following conditions are satisfied:

- $\partial \Omega$ is compact

- there exist $M \in \mathbf{N}$, open sets $W_{1}, \ldots, W_{M} \subseteq \mathbf{R}^{n}$, sets $\Omega_{1}, \ldots, \Omega_{M} \subseteq \mathbf{R}^{n}$, continuous functions $\xi_{1}, \ldots, \xi_{M}: \mathbf{R}^{n-1} \rightarrow \mathbf{R}$ and rigid motions $\mathscr{R}_{1}, \ldots, \mathscr{R}_{M}$ : $\mathbf{R}^{n} \rightarrow \mathbf{R}^{n}$ such that the following conditions hold true:

$$
\begin{aligned}
& -\partial \Omega \subseteq \bigcup_{j=1}^{M} W_{j}, \\
& -\mathscr{R}_{j}\left(\Omega_{j}\right):=\left\{\left(x^{\prime}, x_{n}\right) \in \mathbf{R}^{n-1} \times \mathbf{R}: x_{n}<\xi_{j}\left(x^{\prime}\right)\right\}, \text { for any } j \in\{1, \ldots, M\}, \\
& -W_{j} \cap \Omega=W_{j} \cap \Omega_{j} .
\end{aligned}
$$

Roughly speaking, a domain with continuous boundary is characterized by being locally the hypograph of a continuous function. Notice, however, that a hypograph is not a domain with continuous boundary since its boundary is unbounded, hence the cases presented in Definition 1 and Definition 4 are similar, but conceptually different.

We also remark that Definition 1 and Definition 4 are also valid when $n=1$ (using the standard notation for which $\mathbf{R}^{0}$ reduces to the point $\{0\}$ ).

Remark 5. Simple examples of domains with continuous boundary are the ball and its complement. More generally, if $\Omega$ is a domain with continuous boundary, then so is $\mathscr{C} \Omega$ (indeed, $W_{j}$ may be taken to be the same for both $\Omega$ and $\mathscr{C} \Omega$, while 
the hypograph $\Omega_{j}$ for $\Omega$ becomes $\mathscr{C} \Omega_{j}$ for $\mathscr{C} \Omega$ : notice that $\mathscr{C} \Omega_{j}$ is still a hypograph with respect to an antipodal rotation).

We also point out that not all the continuous, oriented, closed surfaces are boundaries of a domain with continuous boundary, not even continuous closed curves in the plane that are boundaries of open and bounded sets. For instance, the boundary of the Koch snowflake is a closed curve in the plane, but the Koch snowflake itself is not a domain with continuous boundary. Indeed, the Koch snowflake is a bounded set, but it contains infinitely many portions of equilateral triangles. Let $P$ be an accumulation point for these triangles. In any neighborhood of $P$ lie infinitely many sides of the triangles with normal direction of angle of $k \pi / 3$, for any $k \in\{0, \ldots, 6\}$. In particular, if the Koch snowflake were a domain with continuous boundary, then the set $\Omega_{j}$ that contains $P$ would also contains pieces of domains with normal direction of angle of 0 and $2 \pi$ (corresponding to the choices $k=0$ and $k=6$ ). But since these two directions are antipodal, no hypograph can contain both of them, thus proving that the Koch snowflake is not a domain with continuous boundary.

The main density result for domains with continuous boundary goes as follows:

Theorem 6. Let $\Omega$ be an open subset of $\mathbf{R}^{n}$ with continuous boundary. Then, for any $u \in X_{0}^{s, p}(\Omega)$ there exists a sequence $\rho_{\varepsilon} \in C_{0}^{\infty}(\Omega)$ such that $\left\|\rho_{\varepsilon}-u\right\| \rightarrow 0$ as $\varepsilon \rightarrow 0$. In other words, $C_{0}^{\infty}(\Omega)$ is a dense subspace of $X_{0}^{s, p}(\Omega)$.

In the case of the usual fractional Sobolev space $W^{s, p}(\Omega)$, the result given in Theorem 6 has already been mentioned (without a detailed proof) in [18, Theorem 1.4.2.2]. The detailed proof that we present here has a simple structure: to prove Theorem 2 we first translate the function "below", to set its support a bit far from the boundary of the domain, then we mollify the function (to make it smooth), then we cut-off (to make the support compact). Some care is needed to check that any of these actions (translations, mollifications and cut-off) can be performed by paying only a small error in terms of the norm in (1.5).

Then, the proof of Theorem 6 is a modification of the one of Theorem 2: near the boundary, we reduce to the case of the hypograph, thanks to Definition 4. Far from the boundary, one has space enough to mollify without making the support exits the domain. A suitable partition of unity then glues these two features together.

Remark 7. We think that it is an interesting problem to determine the "minimal" regularity assumptions on the domain $\Omega$ under which the density of the smooth functions, compactly supported in $\Omega$, stated in Theorem 6 , holds true. We remark that such property does not hold for any domain $\Omega$, not even when $n=1$, as the following counterexample shows.

Let $\Omega:=(-1,0) \cup(0,1), s \in(1 / 2,1), \psi: \mathbf{R} \rightarrow \mathbf{R}$ be any fixed smooth function supported in $(-1,1)$ with $\psi(0)=1$, and define

$$
\phi(x):= \begin{cases}\psi(x) & \text { if } x \in \Omega, \\ 0 & \text { if } x \notin \Omega .\end{cases}
$$

Then, since integrals disregard sets of measure zero, we have that for any $s \in(0,1)$

$$
\|\phi\|_{H^{s}(\mathbf{R})}=\|\psi\|_{H^{s}(\mathbf{R})}<+\infty
$$

hence $\phi \in H^{s}(\mathbf{R})$. Also, $\phi$ vanishes outside $\Omega$, that is $\phi \in X_{0}^{s, 2}(\Omega)$ by [29, Lemma 7]. 
Now, let $\eta$ be any smooth function supported in $\Omega$. We have that $\eta(0)=0$ and so, denoting by $f:=\phi-\eta$, by the fractional Sobolev embedding (see e.g. [12, Theorem 8.2] and [25, Lemmas 6 and 7]), we obtain that

$$
1=\lim _{\Omega \ni x \rightarrow 0} f(x) \leqslant\|f\|_{L^{\infty}(\Omega)} \leqslant C\|f\|_{H^{s}(\Omega)} \leqslant C\|\phi-\eta\|_{X_{0}^{s, 2}(\Omega)},
$$

where $C$ is a positive constant. Therefore, smooth functions compactly supported in $\Omega$ cannot approximate $\phi$ in $X_{0}^{s, 2}(\Omega)$ (and also in $H^{s}(\Omega)$ ).

Such example easily generalizes in $W^{s, p}(\Omega)$, with $\Omega:=B_{1} \backslash\{0\}$ and $s p>n$, and also in $H^{s}(\Omega)$ for $n \geqslant 2$ and $s>1 / 2$, where $\Omega:=B_{1} \backslash S$, being $S$ a closed segment contained in $\Omega$ (for this one needs to replace the fractional Sobolev embedding with the trace theory, see e.g. [12, Proposition 3.8]).

Remark 8. Sometimes density properties in the fractional framework are similar to the ones in the classical case, as it happens for the results presented here. The reader should not infer that such fractional density properties are always wellexpected. For instance, it turns out that fractional harmonic functions are locally dense in the space of continuous (and even smooth) functions: see [13]. This is in clear contrast with the classical case, since harmonic functions can never approximate a function with a strict maximum (simply, by Maximum Principle!). Hence fractional density properties may be sometimes a very delicate business.

The paper is organized as follows. In the forthcoming Section 2 we state technical and elementary lemmas useful along the paper. In Section 3 we will discuss the case of the hypograph and we will prove Theorem 2. In Section 4 we will consider the case of an open set with continuous boundary and we will give the proof of Theorem 6 . Finally, Appendix A is devoted to the details of the construction of a suitable partition of unity, used in the proof of Theorem 6 .

\section{Some preliminary lemmas}

This section is devoted to the proof of some preliminary lemmas, which will be used in the sequel.

2.1. Supports. We start with some elementary properties of the support of a function.

Lemma 9. Let $u$ and $v: \mathbf{R}^{n} \rightarrow \mathbf{R}$ be two functions. Then,

$$
\operatorname{Supp}(u v) \subseteq(\operatorname{Supp} u) \cap(\operatorname{Supp} v) .
$$

Proof. Let $x \in \operatorname{Supp}(u v)$. We show that $x \in \operatorname{Supp} u$ (the proof that $x \in \operatorname{Supp} v$ is identical).

By construction, there exists a sequence $x_{k} \rightarrow x$ in $\mathbf{R}^{n}$ as $k \rightarrow+\infty$ with $u\left(x_{k}\right) v\left(x_{k}\right) \neq 0$. In particular, we have $u\left(x_{k}\right) \neq 0$ and so $x_{k} \in \operatorname{Supp} u$. Since the support is a closed set, we obtain that $x \in \operatorname{Supp} u$, as desired.

Lemma 10. Let $v: \mathbf{R}^{n} \rightarrow \mathbf{R}$, and $V \subseteq \mathbf{R}^{n}$ be open. Assume that

$$
\operatorname{Supp} v \subseteq V \text {. }
$$

Then, for any $R>0$, there exists $a>0$ such that

$$
B_{R} \cap\left(\operatorname{Supp} v+B_{a}\right) \subseteq V .
$$

The above quantity a depends on $n, v, R$ and $V$. 
Proof. Fix $R>0$. We argue by contradiction, assuming that for any $k \in \mathbf{N}$ there exists

$$
x_{k} \in B_{R} \cap\left(\operatorname{Supp} v+B_{1 / k}\right) \cap(\mathscr{C} V) .
$$

Hence, for any $k \in \mathbf{N}$ there exists $b_{k} \in B_{1 / k}$ and $y_{k} \in \operatorname{Supp} v$ such that $x_{k}=y_{k}+b_{k}$.

We observe that $\left|x_{k}\right| \leqslant R$ for any $k \in \mathbf{N}$, hence, up to a subsequence, we may suppose that

$$
x_{k} \rightarrow P \quad \text { in } \mathbf{R}^{n} \text {, }
$$

for some $P \in \mathbf{R}^{n}$, as $k \rightarrow+\infty$. Moreover,

$$
\left|y_{k}-P\right| \leqslant\left|y_{k}-x_{k}\right|+\left|x_{k}-P\right|=\left|b_{k}\right|+\left|x_{k}-P\right| \rightarrow 0,
$$

hence

$$
y_{k} \rightarrow P \quad \text { in } \mathbf{R}^{n}
$$

as $k \rightarrow+\infty$. We stress that both $\operatorname{Supp} v$ and $\mathscr{C} V$ are closed sets. Hence, since $x_{k} \in$ $\mathscr{C} V$ and $y_{k} \in \operatorname{Supp} v$, we deduce from (2.2) and (2.3) that

$$
P \in(\operatorname{Supp} v) \cap(\mathscr{C} V) \text {. }
$$

Therefore, by (2.1), we obtain

$$
P \in V \cap(\mathscr{C} V)=\varnothing,
$$

that is a contradiction.

The proofs of Theorem 2 and of Theorem 6 are mainly based on a basic technique of convolution (which makes functions $C^{\infty}$ ), joined with a cut-off (which makes their support compact). Here we will give some properties of these operations with respect to the norm in (1.5). Along this section $\Omega$ will denote an open subset of $\mathbf{R}^{n}$.

2.2. Convolution. In the sequel we will denote by $\left|B_{R}\right|$ the Lebesgue measure of the ball $B_{R}$.

Let $\eta \in C_{0}^{\infty}\left(\mathbf{R}^{n}\right)$ be such that $\eta \geqslant 0$ in $\mathbf{R}^{n}$ and Supp $\eta \subseteq B_{1}$. We also assume that $\int_{B_{1}} \eta(x) d x=1$. Also, let $\varepsilon>0$ and let $\eta_{\varepsilon}$ be the mollifier defined as

$$
\eta_{\varepsilon}(x)=\varepsilon^{-n} \eta(x / \varepsilon), \quad x \in \mathbf{R}^{n} .
$$

Finally, for any $u \in X_{0}^{s, p}(\Omega)$ we will denote by $u_{\varepsilon}$ the function defined as the convolution between $u$ and $\eta_{\varepsilon}$, i.e.

$$
u_{\varepsilon}(x)=\left(u * \eta_{\varepsilon}\right)(x), \quad x \in \mathbf{R}^{n} .
$$

Of course, by construction, $u_{\varepsilon}$ is a smooth function, i.e. $u_{\varepsilon} \in C^{\infty}\left(\mathbf{R}^{n}\right)$. On the other hand, if $u$ is supported in $\Omega$ it is not possible, in general, to conclude that $u_{\varepsilon} \in X_{0}^{s, p}(\Omega)$, since the support of $u_{\varepsilon}$ may exceed the one of $u$ and so it may exit $\Omega$.

For small $\varepsilon$, convolutions do not change too much the norm (1.5), according to the following result:

Lemma 11. Let $u \in X^{s, p}\left(\mathbf{R}^{n}\right)$. Then, $\left\|u_{\varepsilon}-u\right\| \rightarrow 0$ as $\varepsilon \rightarrow 0$.

Proof. Since $u \in L^{p}\left(\mathbf{R}^{n}\right)$, by [7, Theorem IV.22] we know that $\left\|u_{\varepsilon}-u\right\|_{L^{p}\left(\mathbf{R}^{n}\right)} \rightarrow 0$ as $\varepsilon \rightarrow 0$. Hence, to get the assertion, it is sufficient to show that

$$
\int_{\mathbf{R}^{n} \times \mathbf{R}^{n}}\left|u_{\varepsilon}(x)-u(x)-u_{\varepsilon}(y)+u(y)\right|^{p} K(x-y) d x d y \rightarrow 0
$$


as $\varepsilon \rightarrow 0$. For this, note that, using the definition of $u_{\varepsilon}$ and the Hölder inequality, we obtain

$$
\begin{aligned}
& \int_{\mathbf{R}^{n} \times \mathbf{R}^{n}}\left|u_{\varepsilon}(x)-u(x)-u_{\varepsilon}(y)+u(y)\right|^{p} K(x-y) d x d y \\
& =\int_{\mathbf{R}^{n} \times \mathbf{R}^{n}} K(x-y)\left|\int_{\mathbf{R}^{n}}(u(x-z)-u(y-z)) \eta_{\varepsilon}(z) d z-u(x)+u(y)\right|^{p} d x d y \\
& =\int_{\mathbf{R}^{n} \times \mathbf{R}^{n}} K(x-y)\left|\varepsilon^{-n} \int_{B_{\varepsilon}}(u(x-z)-u(y-z)) \eta(z / \varepsilon) d z-u(x)+u(y)\right|^{p} d x d y \\
& =\int_{\mathbf{R}^{n} \times \mathbf{R}^{n}} K(x-y)\left|\int_{B_{1}}(u(x-\varepsilon \tilde{z})-u(y-\varepsilon \tilde{z})-u(x)+u(y)) \eta(\tilde{z}) d \tilde{z}\right|^{p} d x d y \\
& \leqslant\left|B_{1}\right|^{p-1} \int_{\mathbf{R}^{n} \times \mathbf{R}^{n}}\left(\int_{B_{1}}|u(x-\varepsilon z)-u(y-\varepsilon z)-u(x)+u(y)|^{p} K(x-y) \eta^{p}(z) d z\right) d x d y \\
& =\left|B_{1}\right|^{p-1} \int_{\left(\mathbf{R}^{n} \times \mathbf{R}^{n}\right) \times B_{1}}|u(x-\varepsilon z)-u(y-\varepsilon z)-u(x)+u(y)|^{p} K(x-y) \eta^{p}(z) d x d y d z .
\end{aligned}
$$

In the last equality we have used both the Tonelli's and Fubini's Theorems. Now, by using the continuity in $L^{p}\left(\mathbf{R}^{n} \times \mathbf{R}^{n}\right)$ of the translations, as in [17, Proposition 8.5], for any $v \in L^{p}\left(\mathbf{R}^{n} \times \mathbf{R}^{n}\right)$ and $w \in \mathbf{R}^{n} \times \mathbf{R}^{n}$ we have

$$
\int_{\mathbf{R}^{n} \times \mathbf{R}^{n}}|v(\widetilde{x}-\varepsilon w)-v(\widetilde{x})|^{p} d \widetilde{x} \rightarrow 0
$$

as $\varepsilon \rightarrow 0$. So, by fixing $z \in B_{1}$ and by choosing $w:=(z, z) \in \mathbf{R}^{n} \times \mathbf{R}^{n}, \widetilde{x}:=(x, y) \in$ $\mathbf{R}^{n} \times \mathbf{R}^{n}$ and

$$
v(x, y):=(u(x)-u(y))(K(x-y))^{1 / p},
$$

which is in $L^{p}\left(\mathbf{R}^{n} \times \mathbf{R}^{n}\right)$ since $u \in X^{s, p}\left(\mathbf{R}^{n}\right)$, we obtain

$$
\int_{\mathbf{R}^{n} \times \mathbf{R}^{n}}|u(x-\varepsilon z)-u(y-\varepsilon z)-u(x)+u(y)|^{p} K(x-y) d x d y \rightarrow 0
$$

as $\varepsilon \rightarrow 0$. Hence,

$$
\psi_{\varepsilon}(z):=\eta^{p}(z) \int_{\mathbf{R}^{n} \times \mathbf{R}^{n}}|u(x-\varepsilon z)-u(y-\varepsilon z)-u(x)+u(y)|^{p} K(x-y) d x d y \rightarrow 0
$$

as $\varepsilon \rightarrow 0$.

Moreover, for a.e. $z \in B_{1}$, taking into account the regularity of $\eta$ and the fact that $u \in X^{s, p}\left(\mathbf{R}^{n}\right)$, we get

$$
\begin{aligned}
\left|\psi_{\varepsilon}(z)\right| \leqslant 2^{p-1} \eta^{p}(z) & \left(\int_{\mathbf{R}^{n} \times \mathbf{R}^{n}}|u(x-\varepsilon z)-u(y-\varepsilon z)|^{p} K(x-y) d x d y\right. \\
& \left.+\int_{\mathbf{R}^{n} \times \mathbf{R}^{n}}|u(x)-u(y)|^{p} K(x-y) d x d y\right) \\
= & 2^{p} \eta^{p}(z) \int_{\mathbf{R}^{n} \times \mathbf{R}^{n}}|u(x)-u(y)|^{p} K(x-y) d x d y \in L^{\infty}\left(B_{1}\right)
\end{aligned}
$$


for any $\varepsilon>0$. Hence, by the Dominated Convergence Theorem we have

$$
\begin{aligned}
& \int_{\left(\mathbf{R}^{n} \times \mathbf{R}^{n}\right) \times B_{1}}|u(x-\varepsilon z)-u(y-\varepsilon z)-u(x)+u(y)|^{p} K(x-y) \eta^{p}(z) d x d y d z \\
& =\int_{B_{1}} \psi_{\varepsilon}(z) d z \rightarrow 0
\end{aligned}
$$

as $\varepsilon \rightarrow 0$. This fact combined with (2.5) gives (2.4), so that the assertion of Lemma 11 is proved.

2.3. Cut-off. In this subsection we will discuss the cut-off technique needed for the density argument. For this, for any $j \in \mathbf{N}$ let $\tau_{j} \in C^{\infty}\left(\mathbf{R}^{n}\right)$ be a function such that

$$
\begin{aligned}
& 0 \leqslant \tau_{j}(x) \leqslant 1 \quad \text { for any } x \in \mathbf{R}^{n}, \\
& \tau_{j}(x)= \begin{cases}1 & \text { if } x \in B_{j}, \\
0 & \text { if } x \in \mathscr{C} B_{j+1},\end{cases}
\end{aligned}
$$

and

$$
\left|\nabla \tau_{j}(x)\right| \leqslant C \quad \text { for any } x \in \mathbf{R}^{n},
$$

where $C$ is a positive constant not depending on $j$. Here, $B_{j}$ denotes the ball centered at 0 with radius $j$.

We have the following result:

Lemma 12. Let $u \in X^{s, p}\left(\mathbf{R}^{n}\right)$. Then, $\operatorname{Supp} \tau_{j} u \subseteq \overline{B_{j+1}} \cap \operatorname{Supp} u$, and $\| \tau_{j} u-$ $u \| \rightarrow 0$ as $j \rightarrow+\infty$.

Proof. The claim on the supports follows from Lemma 9 and (2.7). Now, let us check that

$$
\int_{\mathbf{R}^{n}}\left|\tau_{j}(x) u(x)-u(x)\right|^{p} d x \rightarrow 0
$$

as $j \rightarrow+\infty$. To this goal, we observe that

$$
\left|\tau_{j}(x) u(x)-u(x)\right|^{p}=\left|\tau_{j}(x)-1\right|^{p}|u(x)|^{p} \leqslant 2^{p}|u(x)|^{p} \in L^{1}\left(\mathbf{R}^{n}\right)
$$

by (2.6) and the fact that $u \in L^{p}\left(\mathbf{R}^{n}\right)$. Moreover, thanks to (2.7),

$$
\left|\tau_{j}(x) u(x)-u(x)\right|^{p} \rightarrow 0 \quad \text { a.e. in } \mathbf{R}^{n}
$$

as $j \rightarrow+\infty$. Then, by (2.10), (2.11) and the Dominated Convergence Theorem, we get (2.9).

Now, let us show that

$$
\int_{\mathbf{R}^{n} \times \mathbf{R}^{n}}\left|\tau_{j}(x) u(x)-u(x)-\tau_{j}(y) u(y)+u(y)\right|^{p} K(x-y) d x d y \rightarrow 0
$$

as $j \rightarrow+\infty$. For this, note that

$$
\begin{aligned}
& \left|\tau_{j}(x) u(x)-u(x)-\tau_{j}(y) u(y)+u(y)\right| \\
& =\left|\left(\tau_{j}(x)-1\right)(u(x)-u(y))-\left(\tau_{j}(y)-\tau_{j}(x)\right) u(y)\right|,
\end{aligned}
$$


thus

$$
\begin{aligned}
& \int_{\mathbf{R}^{n} \times \mathbf{R}^{n}}\left|\tau_{j}(x) u(x)-u(x)-\tau_{j}(y) u(y)+u(y)\right|^{p} K(x-y) d x d y \\
& \leqslant 2^{p-1}\left(\int_{\mathbf{R}^{n} \times \mathbf{R}^{n}}\left|\tau_{j}(x)-1\right|^{p}|u(x)-u(y)|^{p} K(x-y) d x d y\right. \\
& \left.\quad+\int_{\mathbf{R}^{n} \times \mathbf{R}^{n}}\left|\tau_{j}(x)-\tau_{j}(y)\right|^{p}|u(y)|^{p} K(x-y) d x d y\right) .
\end{aligned}
$$

Since $u \in X^{s, p}\left(\mathbf{R}^{n}\right)$ and (2.6) holds true, it easily follows that for any $j \in \mathbf{N}$

$$
\left|\tau_{j}(x)-1\right|^{p}|u(x)-u(y)|^{p} K(x-y) \leqslant 2^{p}|u(x)-u(y)|^{p} K(x-y) \in L^{1}\left(\mathbf{R}^{n} \times \mathbf{R}^{n}\right),
$$

while, by (2.7) we deduce that

$$
\left|\tau_{j}(x)-1\right|^{p}|u(x)-u(y)|^{p} K(x-y) \rightarrow 0 \quad \text { a.e. in } \mathbf{R}^{n} \times \mathbf{R}^{n}
$$

as $j \rightarrow+\infty$. Hence, by the Dominated Convergence Theorem, we get

$$
\int_{\mathbf{R}^{n} \times \mathbf{R}^{n}}\left|\tau_{j}(x)-1\right|^{p}|u(x)-u(y)|^{p} K(x-y) d x d y \rightarrow 0
$$

as $j \rightarrow+\infty$.

Also, by (2.6) and (2.8), we have

$$
\begin{aligned}
\left|\tau_{j}(x)-\tau_{j}(y)\right|^{p}|u(y)|^{p} K(x-y) & \leqslant 2^{p}\left\|\tau_{j}\right\|_{C^{1}\left(\mathbf{R}^{n}\right)}^{p} \min \left\{1,|x-y|^{p}\right\}|u(y)|^{p} K(x-y) \\
& =2^{p}(C+1)^{p} m(x-y)|u(y)|^{p} K(x-y),
\end{aligned}
$$

where $m$ is the function defined in (1.3). Since, by changing variables, by (1.3) and the fact that $u \in X^{s, p}\left(\mathbf{R}^{n}\right)$,

$$
\int_{\mathbf{R}^{n} \times \mathbf{R}^{n}} m(x-y) K(x-y)|u(y)|^{p} d x d y=\int_{\mathbf{R}^{n}} m(z) K(z) d z \int_{\mathbf{R}^{n}}|u(y)|^{p} d y<+\infty,
$$

we get that the map

$$
\mathbf{R}^{n} \times \mathbf{R}^{n} \ni(x, y) \mapsto m(x-y) K(x-y)|u(y)|^{p}
$$

belongs to $L^{1}\left(\mathbf{R}^{n} \times \mathbf{R}^{n}\right)$. Thus, by this, (2.15), the fact that

$$
\left|\tau_{j}(x)-\tau_{j}(y)\right|^{p}|u(y)|^{p} K(x-y) \rightarrow 0 \quad \text { a.e. in } \mathbf{R}^{n} \times \mathbf{R}^{n}
$$

as $j \rightarrow+\infty$, and again by the Dominated Convergence Theorem, we obtain

$$
\int_{\mathbf{R}^{n} \times \mathbf{R}^{n}}\left|\tau_{j}(x)-\tau_{j}(y)\right|^{p}|u(y)|^{p} K(x-y) d x d y \rightarrow 0
$$

as $j \rightarrow+\infty$. By (2.13), (2.14) and (2.17) we get (2.12). This and (2.9) end the proof of Lemma 12 .

2.4. Translations. Here we study the effect of the translations on the norm in (1.5). For any $\delta>0$ and any function $u$ we set

$$
u_{\delta}(x):=u\left(x^{\prime}, x_{n}+\delta\right) .
$$

Then we have:

Lemma 13. Let $u \in X^{s, p}\left(\mathbf{R}^{n}\right)$. Then, $\left\|u_{\delta}-u\right\| \rightarrow 0$ as $\delta \rightarrow 0$. 
Proof. We can argue as in the final part of the proof of Lemma 11: we prefer to give a brief explanation, for the reader's convenience.

First of all, the continuity of the translations in $L^{p}\left(\mathbf{R}^{n}\right)$, as in [17, Proposition 8.5], and the fact that $u \in L^{p}\left(\mathbf{R}^{n}\right)$, give that

$$
\int_{\mathbf{R}^{n}}\left|u_{\delta}(x)-u(x)\right|^{p} d x=\int_{\mathbf{R}^{n}}\left|u\left(x^{\prime}, x_{n}+\delta\right)-u\left(x^{\prime}, x_{n}\right)\right|^{p} d x \rightarrow 0
$$

as $\delta \rightarrow 0$, that is

$$
\left\|u_{\delta}-u\right\|_{L^{p}\left(\mathbf{R}^{n}\right)} \rightarrow 0 \quad \text { as } \delta \rightarrow 0 .
$$

Finally, let us show that

$$
\int_{\mathbf{R}^{n} \times \mathbf{R}^{n}}\left|u_{\delta}(x)-u(x)-u_{\delta}(y)+u(y)\right|^{p} K(x-y) d x d y \rightarrow 0
$$

as $\delta \rightarrow 0$. Again the continuity of the translations in $L^{p}\left(\mathbf{R}^{n} \times \mathbf{R}^{n}\right)$ yields that for any $v \in L^{p}\left(\mathbf{R}^{n} \times \mathbf{R}^{n}\right)$ and $w \in \mathbf{R}^{n} \times \mathbf{R}^{n}$

$$
\int_{\mathbf{R}^{n} \times \mathbf{R}^{n}}|v(\widetilde{x}+\delta w)-v(\widetilde{x})|^{p} d \widetilde{x} \rightarrow 0
$$

as $\delta \rightarrow 0$. Here we denote with $e_{n}=(\overline{0}, 1)$, where $\overline{0}=(0, \ldots, 0) \in \mathbf{R}^{n-1}$. Then, by choosing $w:=\left(e_{n}, e_{n}\right) \in \mathbf{R}^{n} \times \mathbf{R}^{n}, \widetilde{x}:=(x, y) \in \mathbf{R}^{n} \times \mathbf{R}^{n}$ and

$$
v(x, y):=(u(x)-u(y))(K(x-y))^{1 / p}
$$

which is in $L^{p}\left(\mathbf{R}^{n} \times \mathbf{R}^{n}\right)$ since $u \in X^{s, p}\left(\mathbf{R}^{n}\right)$, we obtain (2.19). Of course, (2.18) and (2.19) conclude the proof of Lemma 13.

The next lemma says that translations are useful to set the support away from the boundary of a hypograph:

Lemma 14. Let $\Omega$ be a hypograph. Let $u: \mathbf{R}^{n} \rightarrow \mathbf{R}$ be such that ${ }^{1} u=0$ in $\mathscr{C} \Omega$. Then,

$$
\operatorname{Supp} u_{\delta} \subseteq \Omega \text {. }
$$

More precisely, given any $R>0$ there exists $a>0$ such that

$$
B_{R} \cap\left(\operatorname{Supp} u_{\delta}+B_{a}\right) \subseteq \Omega .
$$

The above quantity a only depends on $n, u, \delta, R$ and $\Omega$ (say, $a=a(n, u, \delta, R, \Omega)$ ).

Proof. The proof is an elementary set inclusion, joined with a compactness argument. We provide full details for the convenience of the reader.

First, we prove $(2.20)$. For this, we take $x \in \operatorname{Supp} u_{\delta}$. Then, there exists a sequence of points $x_{k}$ such that $x_{k} \rightarrow x$ in $\mathbf{R}^{n}$ as $k \rightarrow+\infty$ and

$$
0 \neq u_{\delta}\left(x_{k}\right)=u\left(x_{k}^{\prime}, x_{k, n}+\delta\right) .
$$

Therefore, $\left(x_{k}^{\prime}, x_{k, n}+\delta\right) \in \Omega$, since $u=0$ in $\mathscr{C} \Omega$ by assumption. Hence, by Definition 1 , we have that $x_{k, n}+\delta<\xi\left(x_{k}^{\prime}\right)$. Passing to the limit as $k \rightarrow+\infty$ and using

\footnotetext{
${ }^{1}$ We point out that if $u \in X_{0}^{s, p}(\Omega)$ one cannot say that Supp $u \subseteq \bar{\Omega}$ : this is only true by possibly modifying $u$ in a set of measure zero. Indeed, as an example, one can take $\Omega$ to be an open set in $\mathbf{R}^{n}$ and $u$ to be the characteristic function of $\mathbf{Q}^{n}$. In this case, Supp $u=\mathbf{R}^{n}$, but, of course, $u$ is equivalent to the zero function, up to sets of measure zero and so $u \in X_{0}^{s, p}(\Omega)$. This is the reason for which we assume that $u=0$ in $\mathscr{C} \Omega$.
} 
the continuity of $\xi$, we obtain that $x_{n}+\delta \leqslant \xi\left(x^{\prime}\right)$. In particular, since $\delta>0$, we get that $x_{n}<\xi\left(x^{\prime}\right)$ and so $x \in \Omega$. This proves $(2.20)$.

Then, (2.21) follows from (2.20) and Lemma 10.

\section{Proof of Theorem 2 (and of Remark 3)}

Let $\Omega$ be as in Theorem 2 and let $u \in X_{0}^{s, p}(\Omega)$. By possibly changing $u$ in a set of zero measure, we suppose that

$$
u=0 \text { in } \mathscr{C} \Omega \text {. }
$$

Let us fix $\sigma>0$. By Lemma 13 we know that there exists a measurable function $u_{\delta}$ such that

$$
\left\|u_{\delta}-u\right\|<\frac{\sigma}{3}
$$

for $\delta$ sufficiently small, say $\delta \leqslant \bar{\delta}$, with $\bar{\delta}=\bar{\delta}(\sigma)>0$.

Now, let us fix $\delta=\bar{\delta}$ and let $\tau_{j}$ be as in Subsection 2.3. By Lemma 12 we know that

$$
\left\|\tau_{j} u_{\bar{\delta}}-u_{\bar{\delta}}\right\|<\frac{\sigma}{3}
$$

for $j$ large enough, say $j \geqslant \bar{\jmath}$, with $\bar{\jmath}=\bar{\jmath}(\sigma) \in \mathbf{N}$.

For any $\varepsilon>0$ let us consider

$$
\rho_{\varepsilon}:=\tau_{\bar{\jmath}} u_{\bar{\delta}} * \eta_{\varepsilon},
$$

where $\eta_{\varepsilon}$ is the function defined in Subsection 2.2. Of course, $\rho_{\varepsilon} \in C^{\infty}\left(\mathbf{R}^{n}\right)$ by construction. Furthermore, by the standard properties of the convolution (see e.g. [7, Proposition IV.18]) we have that

$$
\operatorname{Supp} \rho_{\varepsilon} \subseteq \operatorname{Supp} \tau_{\bar{\jmath}} u_{\bar{\delta}}+\bar{B}_{\varepsilon} .
$$

On the other hand, by Lemma 12 we have that

$$
\operatorname{Supp} \tau_{\bar{\jmath}} u_{\bar{\delta}} \subseteq \overline{B_{\bar{\jmath}+1}} \cap \operatorname{Supp} u_{\bar{\delta}} .
$$

Now we claim that

$$
\operatorname{Supp} \rho_{\varepsilon} \subseteq B_{\bar{\jmath}+2} \cap\left(\operatorname{Supp} u_{\bar{\delta}}+B_{2 \varepsilon}\right)
$$

if $\varepsilon$ is sufficiently small (possibly in dependence on $\sigma$ ). Indeed: let $P \in \operatorname{Supp} \rho_{\varepsilon}$. Then, by (3.4), there exists $Q \in \operatorname{Supp} \tau_{\bar{\jmath}} u_{\bar{\delta}}$ such that $|P-Q| \leqslant \varepsilon<2 \varepsilon$. Hence, by (3.5), we know that $|Q| \leqslant \bar{\jmath}+1$ and $Q \in \operatorname{Supp} u_{\bar{\delta}}$. In particular, $|P| \leqslant|Q|+|P-Q| \leqslant$ $\bar{\jmath}+1+2 \varepsilon<\bar{\jmath}+2$, if $\varepsilon$ is small enough, thus proving (3.6).

From (2.21) and (3.6), we deduce that Supp $\rho_{\varepsilon}$ is compact and contained in $\Omega$, as long as $\varepsilon$ is small enough, say $2 \varepsilon<a(n, u, \bar{\delta}, \bar{\jmath}+2, \Omega)$ in the notation of Lemma 14 (we also remark that it is possible to use Lemma 14 here in virtue of the normalization performed in (3.1)). As a consequence of this,

$$
\rho_{\varepsilon} \in C_{0}^{\infty}(\Omega),
$$

for $\varepsilon$ small enough.

Furthermore, by Lemma 11

$$
\left\|\rho_{\varepsilon}-\tau_{\bar{\jmath}} u_{\bar{\delta}}\right\|<\frac{\sigma}{3}
$$

for $\varepsilon$ small, say $\varepsilon \leqslant \bar{\varepsilon}$, where $\bar{\varepsilon}=\bar{\varepsilon}(\sigma)>0$. 
Finally, thanks to (3.2), (3.3) and (3.7), we get

$$
\left\|u-\rho_{\varepsilon}\right\| \leqslant\left\|u-u_{\bar{\delta}}\right\|+\left\|u_{\bar{\delta}}-\tau_{\bar{\jmath}} u_{\bar{\delta}}\right\|+\left\|\tau_{\bar{\jmath}} u_{\bar{\delta}}-\rho_{\varepsilon}\right\|<\frac{\sigma}{3}+\frac{\sigma}{3}+\frac{\sigma}{3}=\sigma .
$$

The arbitrariness of $\sigma$ concludes the proof of Theorem 2 .

As for the proof of Remark 3, we argue as follows. By construction

$$
\operatorname{Supp} u_{\bar{\delta}} \subseteq \operatorname{Supp} u+B_{2 \bar{\delta}} \text {. }
$$

This and (3.6) yield that

$$
\operatorname{Supp} \rho_{\varepsilon} \subseteq \operatorname{Supp} u_{\bar{\delta}}+B_{2 \varepsilon} \subseteq \operatorname{Supp} u+B_{2 \bar{\delta}}+B_{2 \varepsilon} \subseteq \operatorname{Supp} u+B_{2(\varepsilon+\bar{\delta})},
$$

thus checking Remark 3.

\section{Proof of Theorem 6}

Roughly speaking, the idea is to use an appropriate partition of unity in order to reduce locally to the case of a hypograph and thus use Theorem 2. Below are the details of the argument.

Let $\Omega$ be as in Theorem 6 . Since $\partial \Omega$ is compact, we may suppose that it is contained in $B_{R}$, for some $R>0$. By possibly replacing $W_{j}$ with $W_{j} \cap B_{R+1}$, we may also suppose that

$$
\text { each } W_{j} \text { is a bounded set contained in } B_{R+1} \text {. }
$$

Hence, the set

$$
W:=W_{1} \cup \cdots \cup W_{M}
$$

is bounded and contained in $B_{R+1}$.

First of all, we claim that

$$
\varrho_{0}:=\inf _{\substack{x \in \mathbf{R}^{n} \backslash W \\ y \in \partial \Omega}}|x-y|>0 .
$$

To prove this, we argue by contradiction and we assume that there exist $x_{j} \in \mathbf{R}^{n} \backslash W$ and $y_{j} \in \partial \Omega$ such that $\left|x_{j}-y_{j}\right| \rightarrow 0$ in $\mathbf{R}$ as $j \rightarrow+\infty$. Since $\partial \Omega$ is compact, we may suppose that $y_{j} \rightarrow y_{\infty} \in \partial \Omega$ as $j \rightarrow+\infty$. Moreover, $\left|x_{j}\right| \leqslant\left|x_{j}-y_{j}\right|+\left|y_{j}\right| \leqslant 1+R$ for large $j$, hence we may also suppose that $x_{j} \rightarrow x_{\infty}$, for some $x_{\infty} \in \mathbf{R}^{n}$. By construction and thanks to the continuity of $|\cdot|$ we get

$$
\left|x_{\infty}-y_{\infty}\right|=\lim _{j \rightarrow+\infty}\left|x_{j}-y_{j}\right|=0,
$$

so that $x_{\infty}=y_{\infty} \in \partial \Omega$. Hence, up to renaming the covering sets, we may suppose that $x_{\infty}=y_{\infty} \in W_{1}$. Since $W_{1}$ is open, we have that both $x_{j}$ and $y_{j}$ must lie in $W_{1}$ for $j$ sufficiently large. But this is in contradiction with the fact that $x_{j} \in \mathbf{R}^{n} \backslash W$, and so (4.3) is proved.

So, we define

$$
W_{M+1}:=\left\{x \in \Omega:|x-y|>\varrho_{0} / 2 \text { for any } y \in \partial \Omega\right\} .
$$

Notice that $W_{M+1}$ may be empty (in such case, one can simply neglect the index $M+1$ from now on). We claim that

$$
\Omega \subseteq \bigcup_{j=1}^{M+1} W_{j} .
$$


To prove it, we argue again by contradiction. Suppose that there exists

$$
x \in \Omega \backslash \bigcup_{j=1}^{M+1} W_{j}
$$

In particular,

$$
x \notin W \text { and } x \notin W_{M+1} .
$$

Then, by the definition of $W_{M+1}$, there exists $y \in \partial \Omega$ such that

$$
|x-y| \leqslant \varrho_{0} / 2 \text {. }
$$

But, also $x \in \mathbf{R}^{n} \backslash W$, so we can use (4.3) and say that $|x-y| \geqslant \varrho_{0}>\varrho_{0} / 2$. This is a contradiction, hence (4.5) is proved.

Now, we take a partition of unity $\left\{\alpha_{j}\right\}_{j \in\{1, \ldots, M+1\}}$ subordinate to the collection of sets $\left\{W_{1}, \ldots, W_{M+1}\right\}$, i.e. $\alpha_{j}: \mathbf{R}^{n} \rightarrow[0,1]$ is smooth, with

$$
\begin{aligned}
\operatorname{Supp} \alpha_{j} & \subseteq W_{j} \text { for } j \in\{1, \ldots, M\}, \\
\operatorname{Supp} \alpha_{M+1} & \subseteq W_{M+1}+B_{\varrho_{0} / 8}
\end{aligned}
$$

and

$$
\sum_{j=1}^{M+1} \alpha_{j}(x)=1 \quad \text { for any } x \in \Omega .
$$

Of course, in this partition of unity, the index $M+1$ must be disregarded when $W_{M+1}=\varnothing$. Since this partition of unity is not completely standard, we give the details of its construction in Appendix A.

Now, we claim that there exists $c>0$ such that, for any $j \in\{1, \ldots, M\}$,

$$
\operatorname{Supp} \alpha_{j}+B_{c} \subseteq W_{j} .
$$

To this purpose, we use (4.6) and Lemma 10 to see that, for any $j \in\{1, \ldots, M\}$, there exists $c_{j} \in(0,1)$ such that

$$
B_{R+2} \cap\left(\operatorname{Supp} \alpha_{j}+B_{c_{j}}\right) \subseteq W_{j} .
$$

Here above, $R$ is the one given in (4.1). Thus we notice that, by (4.6) and (4.1),

$$
\operatorname{Supp} \alpha_{j}+B_{c_{j}} \subseteq W_{j}+B_{c_{j}} \subseteq B_{R+1}+B_{1} \subseteq B_{R+2},
$$

and so

Accordingly, (4.10) becomes

$$
B_{R+2} \cap\left(\operatorname{Supp} \alpha_{j}+B_{c_{j}}\right)=\operatorname{Supp} \alpha_{j}+B_{c_{j}} .
$$

$$
\operatorname{Supp} \alpha_{j}+B_{c_{j}} \subseteq W_{j} .
$$

Since we are dealing with a finite number of indices $j$, we can set $c:=\min \left\{c_{1}, \ldots, c_{M}\right\}$ $>0$ and complete the proof of (4.9).

Now, let $u \in X_{0}^{s, p}(\Omega)$ be as in the statement of Theorem 6. For any $j \in$ $\{1, \ldots, M+1\}$ define

By (4.8), for a.e. $x \in \Omega$

$$
u_{j}:=\alpha_{j} u
$$

$$
\sum_{j=1}^{M+1} u_{j}(x)=\sum_{j=1}^{M+1} \alpha_{j}(x) u(x)=u(x) \sum_{j=1}^{M+1} \alpha_{j}(x)=u(x) .
$$


Moreover, a.e. $x \in \mathscr{C} \Omega$, we get

$$
u(x)=0=u(x) \sum_{j=1}^{M+1} \alpha_{j}(x)=\sum_{j=1}^{M+1} u_{j}(x) .
$$

All in all, for a.e. $x \in \mathbf{R}^{n}$

$$
u(x)=\sum_{j=1}^{M+1} u_{j}(x) .
$$

Now, let us fix $\sigma>0$ and $j \in\{1, \ldots, M\}$. We note that

$$
u_{j} \in X^{s, p}\left(\mathbf{R}^{n}\right)
$$

since so is $u$ (recall that $\alpha_{j}$ is smooth and compactly supported, by (4.6) and (4.1)). Now we check that

$$
u_{j}=0 \text { a.e. in } \mathscr{C} \Omega_{j},
$$

where $\Omega_{j}$ is the set given in Definition 4. To this purpose, we notice that, by the properties of $\Omega_{j}$ and (4.6)

$$
\Omega_{j} \supseteq \Omega_{j} \cap W_{j}=\Omega \cap W_{j} \supseteq \Omega \cap \operatorname{Supp} \alpha_{j} .
$$

As a consequence

$$
\mathscr{C} \Omega_{j} \subseteq \mathscr{C}\left(\Omega \cap \operatorname{Supp} \alpha_{j}\right)=(\mathscr{C} \Omega) \cup\left(\mathscr{C} \operatorname{Supp} \alpha_{j}\right) .
$$

Now, let $x \in \mathscr{C} \Omega_{j}$. By (4.14) we obtain that either $x \in \mathscr{C} \Omega$ (and so $u(x)=0$, up to neglecting a set of zero measure) or $x \in \mathscr{C} \operatorname{Supp} \alpha_{j}$ (and so $\alpha_{j}(x)=0$ ). This says that $u_{j}(x)=\alpha_{j}(x) u(x)=0$ (up to a set of zero measure) and proves (4.13).

By (4.12) and (4.13), we have that $u_{j} \in X_{0}^{s, p}\left(\Omega_{j}\right)$, for any $j \in\{1, \ldots, M\}$. So we can use Theorem 2 and obtain a function

$$
v_{j} \in C_{0}^{\infty}\left(\Omega_{j}\right)
$$

such that

$$
\left\|v_{j}-u_{j}\right\| \leqslant \frac{\sigma}{2 M}
$$

for any $j \in\{1, \ldots, M\}$. Additionally, by Remark 3, we may choose $v_{j}$ such that

$$
\operatorname{Supp} v_{j} \subseteq \operatorname{Supp} u_{j}+B_{c}
$$

where $c>0$ is the positive constant fixed in (4.9). Also, by Lemma 9, we have that $\operatorname{Supp} u_{j} \subseteq \operatorname{Supp} \alpha_{j}$. Using this, (4.9) and (4.17) we obtain

$$
\operatorname{Supp} v_{j} \subseteq \operatorname{Supp} \alpha_{j}+B_{c} \subseteq W_{j} .
$$

More precisely, recalling (4.15), we have

$$
\operatorname{Supp} v_{j} \subseteq W_{j} \cap \Omega_{j}=W_{j} \cap \Omega \subseteq \Omega
$$

and so

$$
v_{j} \in C_{0}^{\infty}(\Omega) .
$$

The reader may appreciate the difference between (4.15) and (4.18).

Now, let us consider $u_{M+1}$. We recall (4.4) and we obtain that

$$
W_{M+1}+B_{\varrho_{0} / 4} \subseteq \Omega \text {. }
$$


So, we define the mollification $u_{M+1, \varepsilon}:=u_{M+1} * \eta_{\varepsilon}$. Using once more the standard properties of the convolution, Lemma 9 and (4.7), we conclude that

$$
\begin{aligned}
\operatorname{Supp} u_{M+1, \varepsilon} & \subseteq \operatorname{Supp} u_{M+1}+\bar{B}_{\varepsilon}=\operatorname{Supp}\left(\alpha_{M+1} u\right)+\bar{B}_{\varepsilon} \\
& \subseteq\left(\operatorname{Supp} \alpha_{M+1} \cap \operatorname{Supp} u\right)+\bar{B}_{\varepsilon} \\
& \subseteq\left(\left(W_{M+1}+B_{\varrho_{0} / 8}\right) \cap \operatorname{Supp} u\right)+\bar{B}_{\varepsilon} \subseteq\left(W_{M+1}+B_{\varrho_{0} / 8}\right)+\bar{B}_{\varepsilon} .
\end{aligned}
$$

Therefore, recalling (4.19), we obtain that

$$
\operatorname{Supp} u_{M+1, \varepsilon} \subseteq \Omega
$$

if $\varepsilon$ is sufficiently small, say $\varepsilon<\varrho_{0} / 8$. Furthermore, by Lemma 11, we have that

$$
\left\|u_{M+1, \varepsilon}-u_{M+1}\right\| \leqslant \frac{\sigma}{4},
$$

as long as $\varepsilon$ is sufficiently small (possibly in dependence of $\sigma$ ).

We stress that (4.20) is not enough to ensure that $u_{M+1, \varepsilon}$ is compactly supported, since $\Omega$ is not necessarily bounded (and neither is $W_{M+1}$ ). So, now we need to perform a further cut-off argument, by defining

$$
u_{M+1, \varepsilon, J}:=\tau_{J} u_{M+1, \varepsilon},
$$

with $\tau_{J}$ as in (2.6). Thus, by Lemma 12 and (4.20), we obtain that

$$
\text { Supp } u_{M+1, \varepsilon, J} \subseteq \overline{B_{J+1}} \cap \operatorname{Supp} u_{M+1, \varepsilon} \subseteq \overline{B_{J+1}} \cap \Omega,
$$

hence

$$
u_{M+1, \varepsilon, J} \in C_{0}^{\infty}(\Omega)
$$

and

$$
\left\|u_{M+1, \varepsilon, J}-u_{M+1, \varepsilon}\right\| \leqslant \frac{\sigma}{4}
$$

if $J$ is large enough (possibly depending on $\sigma$ ). This and (4.21) give that

$$
\left\|u_{M+1, \varepsilon, J}-u_{M+1}\right\| \leqslant \frac{\sigma}{2} \text {. }
$$

Now, we define

$$
\rho_{\sigma}:=\sum_{j=1}^{M} v_{j}+u_{M+1, \varepsilon, J} .
$$

By (4.18) and (4.22) we know that

$$
\rho_{\sigma} \in C_{0}^{\infty}(\Omega) .
$$

Furthermore, using (4.11), (4.16) and (4.23), we conclude that

$$
\begin{aligned}
\left\|u-\rho_{\sigma}\right\| & =\left\|\sum_{j=1}^{M+1} u_{j}-\sum_{j=1}^{M} v_{j}-u_{M+1, \varepsilon, J}\right\| \leqslant \sum_{j=1}^{M}\left\|u_{j}-v_{j}\right\|+\left\|u_{M+1}-u_{M+1, \varepsilon, J}\right\| \\
& \leqslant \sum_{j=1}^{M} \frac{\sigma}{2 M}+\frac{\sigma}{2}=\sigma .
\end{aligned}
$$

The arbitrariness of $\sigma$ ends the proof of Theorem 6 . 


\section{Appendix A. Construction of the partition of unity}

In this appendix, we show how to construct, under the assumption of Theorem 6 , the partition of unity $\left\{\alpha_{j}\right\}_{j \in\{1, \ldots, M+1\}}$ subordinate to the collection of sets $\left\{W_{1}, \ldots\right.$, $\left.W_{M+1}\right\}$ and satisfying conditions (4.6)-(4.8).

To this goal, we set

$$
F:=\overline{(\partial \Omega)+B_{3 \varrho_{0} / 4}},
$$

where $\varrho_{0}$ is the positive constant given in (4.3). Since $\partial \Omega$ is bounded, then so is $F$, that turns out to be compact. Also, we note that

$$
F \subseteq W_{1} \cup \cdots \cup W_{M} .
$$

To prove this, we argue by contradiction: if not, there exists $x_{*} \in F \backslash\left(W_{1} \cup \cdots \cup\right.$ $\left.W_{M}\right)=F \backslash W$. So, since $x_{*} \in F$, there exists a sequence $x_{k} \in(\partial \Omega)+B_{3 \varrho_{0} / 4}$ such that $x_{k} \rightarrow x_{*}$ in $\mathbf{R}^{n}$ as $k \rightarrow+\infty$. Hence, there exist $y_{k} \in \partial \Omega$ and $b_{k} \in B_{3 \varrho_{0} / 4}$ such that $x_{k}=y_{k}+b_{k}$. Up to subsequences, since $\Omega$ is compact, we may suppose that $y_{k} \rightarrow y_{*} \in \partial \Omega$ as $k \rightarrow+\infty$. Thus,

$$
\left|x_{*}-y_{*}\right|=\lim _{k \rightarrow+\infty}\left|x_{k}-y_{k}\right|=\lim _{k \rightarrow+\infty}\left|b_{k}\right| \leqslant \frac{3 \varrho_{0}}{4} .
$$

On the other hand, $x_{*} \in \mathbf{R}^{n} \backslash W$, hence, by (4.3),

$$
\left|x_{*}-y_{*}\right| \geqslant \inf _{\substack{x \in \mathbf{R}^{n} \backslash W \\ y \in \partial \Omega}}|x-y|=\varrho_{0}>\frac{3 \varrho_{0}}{4} .
$$

This is in contradiction with (A.2), and so (A.1) is proved.

Thanks to (A.1) and the fact that $F$ is compact, we can now perform the covering argument used in the proof of [2, Theorem 1.9]. Accordingly, there exist compact sets $C_{j} \subseteq W_{j}$ and functions $\xi_{j} \in C_{0}^{\infty}\left(W_{j}\right)$, with values in $[0,1]$, such that

$$
F \subseteq C_{1} \cup \cdots \cup C_{M}
$$

and

$$
\xi_{j}(x)=1 \text { for all } x \in C_{j}
$$

for any $j=1, \ldots, M$.

Now, let us consider the set $W_{M+1}$ defined in (4.4). We define

$$
\tilde{W}_{M+1}:=W_{M+1}+B_{\varrho_{0} / 16}
$$

and we consider its characteristic function $\chi_{\tilde{W}_{M+1}}$ and its mollification $\xi_{M+1}$ as described in Section 2.2, i.e.

$$
\xi_{M+1}:=\eta_{\varepsilon} * \chi_{\tilde{W}_{M+1}},
$$

for a fixed $\varepsilon$ that we now specify. For this, we notice that $\xi_{M+1}$ is smooth and

$$
\operatorname{Supp} \xi_{M+1} \subseteq \tilde{W}_{M+1}+\overline{B_{\varepsilon}} \subseteq W_{M+1}+B_{\varrho_{0} / 8}
$$

as long as $\varepsilon$ is fixed suitably small, say $\varepsilon<\varrho_{0} / 16$. Furthermore, taking into account this choice of $\varepsilon$ and (A.5), we get for any $x \in W_{M+1}$,

$$
\xi_{M+1}(x)=\int_{B_{\varepsilon}} \chi_{\tilde{W}_{M+1}}(x-y) \eta_{\varepsilon}(y) d y=\int_{B_{\varepsilon}} \eta_{\varepsilon}(y) d y=1 .
$$

Now, we set $\alpha_{1}:=\xi_{1}$ and, recursively,

$$
\alpha_{j}:=\left(1-\xi_{1}\right) \ldots\left(1-\xi_{j-1}\right) \xi_{j},
$$


for any $j \in\{2, \ldots, M+1\}$. By construction, for any $j=1, \ldots, M+1$ the functions $\alpha_{j}$ are smooth, with values in $[0,1]$ and, by Lemma 9 ,

$$
\operatorname{Supp} \alpha_{j} \subseteq \operatorname{Supp} \xi_{j}
$$

which implies (4.6) and (4.7), thanks to the properties of $\xi_{j}$.

It remains to prove (4.8). At this purpose, we claim that, for any $K \in\{1, \ldots$, $M+1\}$

$$
\sum_{j=1}^{K} \alpha_{j}=1-\left(1-\xi_{1}\right) \ldots\left(1-\xi_{K}\right)
$$

This can be checked by induction. Indeed, (A.7) is obvious when $K=1$. Now, suppose that (A.7) holds for some $K$ : then

$$
\begin{aligned}
\sum_{j=1}^{K+1} \alpha_{j} & =1-\left(1-\xi_{1}\right) \ldots\left(1-\xi_{K}\right)+\alpha_{K+1} \\
& =1-\left(1-\xi_{1}\right) \ldots\left(1-\xi_{K}\right)+\left(1-\xi_{1}\right) \ldots\left(1-\xi_{K}\right) \xi_{K+1} \\
& =1-\left(1-\xi_{1}\right) \ldots\left(1-\xi_{K}\right)\left(1-\xi_{K+1}\right),
\end{aligned}
$$

thus completing the inductive step and proving (A.7).

With this, we can check (4.8) by arguing as follows. Fix $x \in \Omega$. If $x \in F$, we use (A.3) to see that $x \in C_{j_{o}}$ for some $j_{o} \in\{1, \ldots, M\}$. So, by (A.4), we get that $\xi_{j_{o}}(x)=1$ and therefore

$$
\left(1-\xi_{1}(x)\right) \ldots\left(1-\xi_{M+1}(x)\right)=0 .
$$

Consequently, by (A.7), we see that

$$
\sum_{j=1}^{M+1} \alpha_{j}(x)=1-\left(1-\xi_{1}(x)\right) \ldots\left(1-\xi_{M+1}(x)\right)=1 .
$$

This proves (4.8) when $x \in F$.

Finally, we check (4.8) when $x \in \Omega \backslash F$. For this, we notice that, in this case,

$$
|x-y| \geqslant \frac{3 \varrho_{0}}{4} \text { for any } y \in \partial \Omega
$$

Indeed, if (A.8) were false, there would exist $y \in \partial \Omega$ such that $|x-y|<3 \varrho_{0} / 4$. So one sets $b:=x-y \in B_{3 \varrho_{0} / 4}$ and obtains that $x=y+b \in(\partial \Omega)+B_{3 \varrho_{0} / 4} \subseteq F$, against our assumption. This proves (A.8).

In turn, (A.8) and (4.4) imply that $x \in W_{M+1}$, and therefore, by (A.6), we obtain that $\xi_{M+1}(x)=1$. Hence, by (A.7), we conclude that

$$
\sum_{j=1}^{M+1} \alpha_{j}(x)=1-\left(1-\xi_{1}(x)\right) \ldots\left(1-\xi_{M+1}(x)\right)=1 .
$$

This proves (4.8) also when $x \in \Omega \backslash F$, and so the construction of the desired partition of unity is complete. 


\section{References}

[1] Adams, R. A.: Sobolev spaces. - Academic Press, New York, 1975.

[2] Agmon, S.: Lectures on elliptic boundary value problems. - Mathematical Studies 2, D. Van Nostrand Co., Inc., Princeton, N.J.-Toronto-London, 1965.

[3] Autuori, G., and P. Pucci: Elliptic problems involving the fractional Laplacian in $\mathbf{R}^{N}$. - J. Differential Equations 255:8, 2013, 2340-2362.

[4] Autuori, G., and P. Pucci: Existence of entire solutions for a class of quasilinear elliptic equations. - NoDEA Nonlinear Differential Equations Appl. 20:3, 2013, 977-1009.

[5] Barles, G., E. Chasseigne, and C. Imbert: On the Dirichlet problem for second-order elliptic integro-differential equations. - Indiana Univ. Math. J. 57:1, 2008, 213-246.

[6] Barrios, B., E. Colorado, A. De Pablo, and U. Sanchez: On some critical problems for the fractional Laplacian operator. - J. Differential Equations 252, 2012, 6133-6162.

[7] Brezis, H.: Analyse fonctionelle. Théorie et applications. - Masson, Paris, 1983.

[8] CABré, X., and J. TAN: Positive solutions of nonlinear problems involving the square root of the Laplacian. - Adv. Math. 224:5, 2010, 2052-2093.

[9] Caffarelli, L., and L. Silvestre: An extension problem related to the fractional Laplacian. - Comm. Partial Differential Equations 32:7-9, 2007, 1245-1260.

[10] Caffarelli, L., and L. Silvestre: Regularity theory for fully nonlinear integro-differential equations. - Comm. Pure Appl. Math. 62:5, 2009, 597-638.

[11] Capella, A.: Solutions of a pure critical exponent problem involving the half-Laplacian in annular-shaped domains. - Commun. Pure Appl. Anal. 10:6, 2011, 1645-1662.

[12] Di Nezza, E., G. Palatucci, and E. Valdinoci: Hitchhiker's guide to the fractional Sobolev spaces. - Bull. Sci. Math. 136:5, 2012, 521-573.

[13] Dipierro, S., O. Savin, and E. Valdinoci: All functions are locally $s$-harmonic up to a small error. - Preprint, http://arxiv.org/pdf/1404.3652v1.pdf.

[14] Dong, H., and D. KIm: On $L_{p}$-estimates for a class of non-local elliptic equations. - J. Funct. Anal. 262:3, 2012, 1166-1199.

[15] Felmer, P., A. QuaAs, and J. Tan: Positive solutions of nonlinear Schrödinger equation with the fractional Laplacian. - Proc. Roy. Soc. Edinburgh Sect. A 142:6, 2012, 1237-1262.

[16] Fiscella, A.: Saddle point solutions for non-local elliptic operators. - Topol. Methods Nonlinear Anal. (to appear).

[17] Folland, G. B.: Real analysis. Modern techniques and their applications. - J. Wiley, New York, 1984.

[18] Grisvard, P.: Elliptic problems in nonsmooth domains. - Pitman, Boston, 1985.

[19] McLean, W.: Strongly elliptic systems and boundary integral equations. - Cambridge Univ. Press, Cambridge, 2000.

[20] Molica Bisci, G.: Fractional equations with bounded primitive. - Appl. Math. Lett. 27, 2014, $53-58$.

[21] Molica Bisci, G., and R. Servadei: A bifurcation result for nonlocal fractional equations. - Anal. Appl. (to appear).

[22] Ros-Oton, X., and J. Serra: Fractional Laplacian: Pohozaev identity and nonexistence results. - C. R. Math. Acad. Sci. Paris 350:9-10, 2012, 505-508.

[23] Ros-Oton, X. and J. Serra: The Pohozaev identity for the fractional Laplacian. - Arch. Ration. Mech. Anal. 213:2, 2014, 587-628. 
[24] Servadei, R.: The Yamabe equation in a non-local setting. - Adv. Nonlinear Anal. 2, 2013, $235-270$.

[25] Servadei, R., and E. Valdinoci: Mountain pass solutions for non-local elliptic operators. J. Math. Anal. Appl. 389, 2012, 887-898.

[26] Servadei, R., and E. VAldinoci: Variational methods for non-local operators of elliptic type. - Discrete Contin. Dyn. Syst. 33:5, 2013, 2105-2137.

[27] Servadei, R., and E. Valdinoci: A Brezis-Nirenberg result for non-local critical equations in low dimension. - Commun. Pure Appl. Anal. 12:6, 2013, 2445-2464.

[28] Servadei, R., and E. Valdinoci: On the spectrum of two different fractional operators. Proc. Roy. Soc. Edinburgh Sect. A 144, 2014, 831-855.

[29] Servadei, R., and E. Valdinoci: The Brezis-Nirenberg result for the fractional Laplacian. Trans. Amer. Math. Soc. (to appear).

[30] Servadei, R., and E. Valdinoci: Fractional Laplacian equations with critical Sobolev exponent. - Preprint, http://www.math.utexas.edu/mp_arc-bin/mpa?yn=12-58.

[31] Silvestre, L.: Regularity of the obstacle problem for a fractional power of the Laplace operator. - PhD Thesis, University of Texas at Austin, http://math.uchicago.edu/ luis/ preprints/luisdissreadable.pdf, 2005.

[32] Stinga, P.R., and Torrea, J. L.: Extension problem and Harnack's inequality for some fractional operators. - Commun. Partial Differ. Equations 35:10-12, 2010, 2092-2122.

[33] TAN, J.: The Brezis-Nirenberg type problem involving the square root of the Laplacian. Calc. Var. Partial Differential Equations 36:1-2, 2011, 21-41.

Received 21 May 2014 • Accepted 4 August 2014 